\title{
Philosophiques
}

\section{Commentaire sur Delisle. Les philosophies du néo-darwinisme}

\section{Michel Morange}

Volume 38, numéro 1, printemps 2011

Les conférences Hugues Leblanc 2010

URI : https://id.erudit.org/iderudit/1005729ar

DOI : https://doi.org/10.7202/1005729ar

Aller au sommaire du numéro

Éditeur(s)

Société de philosophie du Québec

ISSN

0316-2923 (imprimé)

1492-1391 (numérique)

Découvrir la revue

Citer ce document

Morange, M. (2011). Commentaire sur Delisle. Les philosophies du néo-darwinisme. Philosophiques, 38(1), 281-284.

https://doi.org/10.7202/1005729ar d'utilisation que vous pouvez consulter en ligne.

https://apropos.erudit.org/fr/usagers/politique-dutilisation/ 


\title{
Commentaire sur Delisle. Les philosophies du néo-darwinisme
}

\author{
MICHEL MORANGE \\ Ens et Université Paris 6 \\ morange@biologie.ens.fr
}

Dans son ouvrage, Richard Delisle développe une étude minutieuse des épistémologies et des ontologies implicites dans le travail de cinq des principaux fondateurs de la théorie actuelle de l'évolution. Cette dernière, qui s'est progressivement imposée entre les années 1940 et 1960, est désignée par les termes de "Synthèse moderne".

Le résultat de cette étude est étonnant, révélant la présence, chez les fondateurs de cette théorie, de visions bien différentes du consensus actuel qui ramène l'explication de l'ensemble des phénomènes évolutifs dans le monde vivant au mécanisme de variation/sélection.

À la lecture de Richard Delisle, la célèbre phrase de Theodosius Dobzhansky «Rien n'a de sens en biologie, si ce n'est au regard de l'évolution " prend un sens différent. Il ne s'agit pas seulement de constater que les êtres vivants sont le fruit d'une histoire évolutive, mais de faire comprendre que les caractéristiques des organismes ne peuvent être expliquées que par la place qu'ils occupent dans un processus évolutif qui a commencé dans le monde inanimé, et est encore appelé à se poursuivre.

L'ouvrage de Richard Delisle est utile, car il lutte contre cette "pensée unique» qui a gagné le champ des travaux sur l'évolution. La pluralité de points de vue a disparu aujourd'hui, en grande partie à cause de l'effet unificateur de la Synthèse moderne, et la vision "anhistorique ", horizontale comme la désigne Richard Delisle, de Mayr s'est imposée.

Cette vision l'a emporté, non pas parce que les arguments de Huxley et de Dobzhansky en faveur d'une évolution orientée ont été réfutés, mais parce que, simplement, ils ont été "poussés sous le tapis ». Je souscris pleinement au point de vue de Richard Delisle, qui reflète celui de plusieurs des fondateurs de la Synthèse moderne: la description actuelle du mécanisme de variation/sélection n'est pas suffisante pour rendre compte de l'évolution des organismes en tant que phénomène global.

Comment aller au-delà, et enrichir la théorie actuelle de l'évolution? Richard Delisle n'est pas très explicite, mais il semble privilégier l'élaboration d'un nouveau modèle d'évolution cosmique, mieux construit, moins flou que ceux d'Huxley et de Dobzhansky. Il fait remarquer que rien ne prouve que la science de demain privilégiera autant que celle d'aujourd'hui les mécanismes, et refusera toute place aux causes finales. La méthode scientifique est seulement définie par l'histoire, et elle s'est beaucoup transformée depuis la naissance de la science moderne. 
Personnellement, j'ai beaucoup de mal cependant à voir comment une théorie de l'évolution cosmique viendrait expliquer l'évolution des formes vivantes. Parler d'un stade pré-paradigmatique pour la théorie actuelle de l'évolution me semble incorrect. Cette discordance d'opinions que Richard Delisle décrit dans les années 1940-1960 pourrait être plutôt interprétée comme caractéristique de la période révolutionnaire qui a précédé l'installation du paradigme de la Synthèse moderne. Parmi les évolutionnistes, les opinions divergentes sont devenues rares. S'il y a aujourd'hui débat, c'est entre les biologistes de l'évolution et les biologistes fonctionnalistes: ce qui est une explication suffisante pour les premiers - le schéma néo-darwinien — ne l'est pas pour les seconds.

L'autonomie du monde vivant, l'importance des mécanismes dans les explications biologiques, et la place mineure réservée aux lois, semblent être des acquis, auxquels il sera difficile de renoncer. Un abandon de la recherche des causes efficientes pour celle des causes finales me semble également bien peu probable! L'observation des progrès «évidents» des organismes n'a guère de poids devant un mécanisme causal à la vertu explicative largement démontrée. Si la méthode scientifique évolue, ses transformations n'en sont pas pour autant réversibles! Elles ne sont pas plus réversibles que celles des formes vivantes. L'abandon des causes finales me semble être un phénomène irréversible, de même que l'idée qu'il existe des schèmes explicatifs spécifiques du monde vivant est un acquis définitif. Je ne vois pas, dans l'hypothèse avancée par Richard Delisle, comment le basculement de la théorie actuelle de l'évolution vers une théorie de l'évolution cosmique pourrait s'opérer. Il y a, à l'heure actuelle, un écart infranchissable entre l'observation vague de tendances progressives dans l'évolution, et la possibilité de proposer des processus causaux pour les expliquer.

Si une transformation de la théorie évolutive est nécessaire, ce dont je suis autant convaincu que Richard Delisle, s'il est envisageable d'expliquer l'évolution des formes vivantes et non pas seulement, comme c'est le cas à l'heure actuelle, de rendre compte de la possibilité de cette évolution, ce changement ne pourra venir que d'un enrichissement des mécanismes à l'origine de l'évolution des formes vivantes, et non de leur abandon au profit d'une explication alternative de nature totalement différente.

Il me semble aussi évident que ces transformations n'impliqueront pas le retour à l'idée que l'évolution a un sens, mais permettront simplement un dessin plus précis des multiples orientations accessibles à l'évolution des organismes. Il s'agira de réinsuffler une dimension historique dans l'évolution du monde vivant, sans pour autant revenir à un déterminisme historique dépassé. La part de hasard et de contingence que le darwinisme a introduite dans l'évolution du monde vivant n'est pas près de disparaître.

Une caractéristique souvent peu discutée de la théorie actuelle de l'évolution est sa nature abstraite. Ce que nous entendons par là est que les mécanismes proposés sont indépendants de la nature des objets auxquels ils 
s'appliquent. C'est un peu comme si un chimiste espérait expliquer les réactions qu'il observe en ignorant tout des atomes qui forment les molécules. Le mécanisme néo-darwinien demande à être enrichi par la prise en compte de "ce» sur quoi opère la variation/sélection. Cet enrichissement peut s'opérer à deux niveaux; d'abord, à celui de la variation, restée jusqu'à aujourd'hui le parent pauvre de la théorie darwinienne - au contraire du processus de sélection qui a reçu beaucoup plus d'attention. La nature précise des variations n'est pas indifférente pour comprendre l'action ultérieure de la sélection naturelle, même si elle n'oriente pas celle-ci dans une direction particulière. Une duplication de gène ou de génome n'ouvre pas le même champ des possibles que la variation ponctuelle d'une base dans la molécule d'ADN.

Le deuxième niveau est celui des relations entre les variations génétiques, et leurs effets sur l'organisme. Quand la Synthèse moderne fut développée, il était déjà bien connu que les gènes interagissaient entre eux, et que le phénotype n'était pas la somme des effets phénotypiques de chaque gène. Grâce aux résultats obtenus par la biologie moléculaire et la biologie cellulaire, la nature de ces interactions est aujourd'hui bien mieux connue, et la longue chaîne causale qui relie le génotype et le phénotype mieux décrite.

À ces deux niveaux, la transformation évolutive des organismes change en permanence les règles du jeu. En particulier, les relations entre les gènes et le phénotype ont pris des formes différentes, et nouvelles.

Il ne s'agit donc pas de renoncer à l'actualisme, et à la pérennité des mécanismes fondamentaux de l'évolution. Ce qui change, ce sont les "objets" sur lesquels jouent ces mécanismes. Ces objets sont eux-mêmes le produit de l'action de ces mécanismes, et leurs transformations en sont le résultat. Il s'agit d'une émergence diachronique.

En proposant que l'avenir de la théorie de l'évolution, et (peut-être) la résolution des conflits si bien décrits par Richard Delisle résident dans cette description précise, je ne suis pas très original. C'est déjà la voie dessinée par Marc Kirschner et John Gerhart ${ }^{1}$. Surtout, c'est le choix, au moins implicite, que font beaucoup de chercheurs en tentant de rapprocher biologie évolutive et biologie fonctionnelle, en substituant au cadre jusqu'alors vide du mécanisme néo-darwinien les mécanismes précis de variation/sélection à l'origine des phénomènes évolutifs. Ces recherches peuvent porter sur des systèmes «naturels » - les travaux se concentrent aujourd'hui sur des phénomènes simples comme l'évolution des structures et des fonctions protéiques -, ou des systèmes "artificiels ", comme dans les essais d'évolution expérimentale in vitro.

Il est difficile de savoir ce qui «sortira» de ce rapprochement. Les difficultés sont immenses, et les résultats resteront sans doute modestes encore longtemps. Je serais cependant prêt à parier, au vu des «surprises » qui ont

1. M. W. Kirschner and J. C. Gerhart, The Plausibility of Life: Resolving Darwin's Dilemma New Haven, Yale University Press, 2005. 
déjà émergé de ces travaux ${ }^{2}$, que croîtra rapidement l'idée qu'il reste encore beaucoup à découvrir, et donc à expliquer, concernant l'évolution des formes vivantes.

2. J. E. Barrick, D. S. Yu, S. H. Yoon, H. Jeong, T. K. Oh, D. Schneider, R. E. Lenski and J. Kim; F. "Genome Evolution and Adaptation in a Long-Term Experiment with Escherichia coli» Nature 461, 2009, 1243-1247. 